\title{
A NOTE ON PATTERSON MEASURES
}

\author{
Kurt Falk and PekKa Tukia
}

\begin{abstract}
Conformal measures are measures satisfying a certain transformation rule for elements of a Kleinian group $G$ and are normally supported by the limit set of $G$. They are usually constructed by a method due to S. J. Patterson as weak limits of measures supported by a fixed orbit of $G$ in the hyperbolic space, often identified with the unit ball $\mathbf{B}^{n}$. We call such limit measures Patterson measures. This has been the predominant way to obtain conformal measures and one may get the impression that all conformal measures are Patterson measures. We show in this note that this is not the case and two concrete examples are given in the last section.
\end{abstract}

\section{Introduction}

If $G$ is a group of Möbius transformations on the closed unit ball $\overline{\mathbf{B}^{n}}$, then a conformal measure of dimension $\delta$ for $G$ is a real-valued, non-negative, finite Borel measure $\mu$ on $\overline{\mathbf{B}^{n}}$ such that

$$
\mu(g A)=\int_{A}\left|g^{\prime}\right|^{\delta} d \mu
$$

for measurable $A \subset \overline{\mathbf{B}^{n}}$ and $g \in G$. Usually, $G$ is Kleinian and one is interested in conformal measures supported by the limit set $L(G)$ of $G$ with $\delta$ being the exponent of convergence $\delta_{G}$ of $G$. Patterson $[\mathrm{P}]$ constructed such measures if $G$ is a Fuchsian group and this construction was later generalized by Sullivan $[\mathrm{S}]$ to the situation of Kleinian groups.

Patterson's method was to construct such measures as weak limits of probability measures $\mu_{i}$ supported by an orbit $G z$ of $G$ in $\mathbf{B}^{n}$. The measures $\mu_{i}$ are constructed so that, given any neighborhood $U$ in $\overline{\mathbf{B}^{n}}$ of the limit set, the $\mu_{i}$-mass of the complement of $U$ tends to 0 as $i \rightarrow \infty$. The measures $\mu_{i}$ are constructed using the Poincaré series of $G$ defined for $y, z \in \mathbf{B}^{n}$ as

$$
P_{\delta}(y, z)=\sum_{g \in G} e^{-\delta d(y, g(z))} .
$$

This series converges if $\delta>\delta_{G}$ and diverges if $\delta<\delta_{G}$. If $\delta=\delta_{G}$, then the series may diverge or converge; if it diverges, $G$ is of divergence type (at the exponent

Received June 14, 2005; revised November 18, 2005. 
of convergence). In the divergence case, the construction of $\mu_{i}$ is very simple: One lets $\mu_{i}$ be a weighted sum of atomic measures $\mu_{g(z)}$ with total mass 1 concentrated at $g(z)$. One sets

$$
\mu_{i}=c_{i} \sum_{g \in G} e^{-\delta_{i} d(y, g(z))} \mu_{g(z)}
$$

where $\delta_{i}>\delta_{G}$ and $\delta_{i} \rightarrow \delta_{G}$ as $i \rightarrow \infty$. The constant $c_{i}$ is $P_{\delta_{i}}(y, z)^{-1}$, and is chosen so that the total mass is 1 . If the Poincare series diverges at the exponent of convergence, a subsequence of $\mu_{i}$ 's will have a weak limit which is a conformal measure on the limit set. The reason why the limit measure is conformal is that the ratio of $\left|g^{\prime}(0)\right|$ to $e^{-d(0, g(0))}$ tends to 1 as $d(0, g(0)) \rightarrow \infty$ which easily implies the conformality of the limit if $z=y=0$. Note that a weak limit of (3) is conformal, meaning that (1) holds true, only if $y=0$. In all other cases one needs to slightly reformulate the definition of conformality as done in $[\mathrm{N}]$.

Unfortunately, this simple strategy does not work if the Poincaré series is of convergence type. Patterson $[\mathrm{P}]$ overcame this difficulty by modifying the Poincaré series so that it still diverges if $\delta<\delta_{G}$ and converges if $\delta>\delta_{G}$, but if $\delta=\delta_{G}$ it necessarily diverges. The modified Poincare series is obtained by using a real function $h(t)$ defined for non-negative $t$. We set

$$
P_{h, \delta}(y, z)=\sum_{g \in G} h(d(y, g(z))) e^{-\delta d(y, g(z))}
$$

and call $P_{h, \delta}$ the $h$-modified Poincaré series for $G$. The function $h$ has the property that $h(t) \rightarrow \infty$ as $t \rightarrow \infty$ in such a way that we obtain divergence for $\delta=\delta_{G}$ but that otherwise the convergence is as for $P_{\delta}$. One can choose $h$ so that if one replaces in $(3) e^{-\delta_{i} d(y, g(z))}$ by $h(d(y, g(z))) e^{-\delta i d(y, g(z))}$, then a subsequence has a weak limit which is a conformal measure if $y=0$. The map $h$ needs to satisfy certain conditions in order for this process to work; these are the conditions $1^{\circ}-3^{\circ}$ to be discussed later.

If the group is of the first kind, then the Lebesgue measure on the boundary sphere $\mathbf{S}^{n-1}$ of hyperbolic space is a conformal measure. If the group is of the second kind, giving a conformal measure on the limit set is a non-trivial problem. Patterson's construction seems to have been the only one to give measures on the limit set at the exponent of convergence. For instance, Nicholls' book [N] discusses only Patterson's method to construct conformal measures and one easily gets the impression that all conformal measures on the limit set can be obtained by this construction.

The purpose of this note is to show that there are conformal measures which cannot be obtained by the Patterson construction even if the dimension of the measure is the exponent of convergence. Our argument applies if the Poincare series of $G$ converges at the exponent of convergence and is based on the analysis of ends and end limit points of $G$; these are defined in the next section. If $E$ is an end of $G$, then there is an endgroup $G_{E}$ associated to $E$ as well as a subset of $L(G)$ called the end limit set of $E$ and denoted by $L_{e}(E)$. If $\delta_{G_{E}}<\delta_{G}$, then 
we will show that any Patterson measure vanishes on the end limit point set of $E$. On the other hand, we know that if $L_{e}(E) \neq \emptyset$, then the union of $g\left(L_{e}(E)\right)$, $g \in G$, supports a conformal measure $\mu_{E}$ for $G$ of dimension $\delta_{G}$, cf. [AFT, Theorem 4.4]. (We say that a subset of $\overline{\mathbf{B}^{n}}$ supports a measure if its complement has measure zero.) Thus $\mu_{E}$ cannot be a Patterson measure for $G$. More generally, this result is still valid even when $\delta_{G_{E}}=\delta_{G}$, but the $h$-modified Poincaré series for $G_{E}$ converges at the exponent $\delta_{G}$. This follows from our Theorem in Section 5 .

The method used in [AFT] to construct measures supported by the end limit points was to take a sequence of points $z_{i}$ contained in the end and exiting to the end in the sense that if $C \subset \mathbf{B}^{n}$ is compact, then the $z_{i}$ eventually escape from $G C$. If the Poincare series of $G$ converges at $\delta$, then there is a conformal measure of total mass 1 supported by the $G_{E}$-orbit of $z_{i}$ where $G_{E}$ is the stabilizer of the end. These measures have a weak limit which is supported by the end limit points of $E$ and this limit measure can be extended to a conformal measure of $G$. This is the only alternative construction of conformal measures, in addition to the Patterson construction, of which we know.

In Section 6 we shall give two examples of conformal measures of dimension equal to the exponent of convergence which cannot be obtained by the Patterson construction. The first example of an atomic measure on the orbit of a parabolic fixed point of a convergence group was one of the main motivations for this work. The second example is more complex in that it combines a geometrically infinite, topologically tame Kleinian group of the second kind acting in hyperbolic 3-space with an infinitely generated group with exponent of convergence strictly less than 2. The latter gives an end with boundary, as defined below, for the associated hyperbolic manifold. The corresponding end limit set then supports a conformal measure which, by our main result, cannot be a Patterson measure.

\section{Definitions and notations}

In this paper $G$ is a discrete group of Möbius transformations of the closed euclidean unit ball $\overline{\mathbf{B}^{n}}$. The limit set is denoted $L(G)$. We let $M_{G}=\mathbf{B}^{n} / G$ and $\bar{M}_{G}=\left(\overline{\mathbf{B}^{n}} \backslash L(G)\right) / G$ so that $M_{G} \subset \bar{M}_{G}$. We denote by $\partial A$ the boundary in $M_{G}$ for subsets of $M_{G}$, and by $\bar{\partial} A$ the boundary in $\bar{M}_{G}$ for subsets of $\bar{M}_{G}$.

An end of $M_{G}$ is an open connected subset $E$ of $M_{G}$ with non-compact closure in $M_{G}$ and such that $\partial E$ is compact and non-empty. We will also consider ends with boundary in $\bar{M}_{G}$; an open subset $E$ of $\bar{M}_{G}$ is called an end with boundary if $E$ is connected, has non-compact closure in $\bar{M}_{G}$ but $\bar{\partial} E$ is compact and non-empty.

We will also consider lifts of ends to the $n$-ball. Let $\pi: \overline{\mathbf{B}^{n}} \backslash L(G) \rightarrow \bar{M}_{G}$ be the canonical projection. An end [with boundary] of $G$ is a connected component of $\pi^{-1}(E)$ where $E$ is an end of $M_{G}$ [or of $\bar{M}_{G}$ if $E$ is an end with boundary]. Thus an end of $G$ refers to subsets of $\mathbf{B}^{n}$ or of $\overline{\mathbf{B}^{n}} \backslash L(G)$ whereas an end of $M_{G}$ or of $\bar{M}_{G}$ lives in the quotient. 
If $E$ is an end of $G$, possibly with boundary, then its set of endpoints is the set of points $z \in S^{n-1}$ such that if $R$ is a hyperbolic ray towards $z$, then $R$ has a subray $R^{\prime}$ contained in the end such that the hyperbolic distance $d(u, \partial E) \rightarrow \infty$ as $u$ moves towards $z$ on $R^{\prime}$; here $\partial$ refers to the boundary in $\mathbf{B}^{n}$. An endpoint $z$ of $E$ is an end limit point of $E$ if $z$ is also a limit point. Note that if $G_{E}$ is the stabilizer of $E$ in $G$ and $z$ is an endpoint of $E$, then $z \in L\left(G_{E}\right)$ as soon as $z \in L(G)$. In other words, every end limit point of $E$ is contained in $L\left(G_{E}\right)$. This follows from Lemma 3.1 of $[\mathrm{AFT}]$ which is valid also for ends with boundary.

The set of end limit points of $E$ is denoted by $L_{e}(E)$. Note that there may be two ends with the same end group. This happens if $G$ is a finitely generated, doubly degenerate Kleinian surface group acting on $\mathbf{B}^{3}$ (see for instance $[\mathrm{MT}]$ for standard definitions).

The notion of a bounded end is crucial for one of our examples. Let $E$ be an end of $M_{G}$ and $F$ be a component of the lift of $E$ to $\mathbf{B}^{n}$. As before, let $G_{F}$ denote the stabilizer of $F$ in $G$. Since $g F=F$ for $g \in G_{F}$, and $g F \cap F=\emptyset$ if $g \in G \backslash G_{F}$, we can identify $E=F / G$ and $F / G_{F}$. We can say now that the end $E$ (or its lift $F$ ) is a bounded end if $\bar{M}_{G_{F}} \backslash E$ is compact. The definition is similar for ends with boundary, with $\mathbf{B}^{n}$ replaced by $\overline{\mathbf{B}^{n}} \backslash L(G)$.

\section{The Patterson construction}

We will now discuss Patterson's construction to the extent needed in this paper. We first note that sometimes one replaces $h(d(y, g(z)))$ in the formula (4) by $h\left(e^{d(y, g(z))}\right)$, for instance this is so in Nicholls' book [N] which we use as our reference. The conditions $1^{\circ}$ and $2^{\circ}$ below correspond to Nicholls' conditions 1 and 2 in Lemma 3.1.1 with this modification. Thus if $h_{N}$ is as in [N], we need to set $h(t)=h_{N}\left(e^{t}\right)$ in order to get our $h$. The function $h$ used in (4) needs to be continuous and non-decreasing and in addition it must satisfy

$1^{\circ}$. $P_{h, \delta}$ converges for $\delta>\delta_{G}$ and diverges for $\delta \leq \delta_{G}$, and

$2^{\circ}$. For any $\varepsilon>0$ there exists $r_{0}$ such that if $r>r_{0}$, then $h(t+r) \leq e^{\varepsilon t} h(r)$.

This is all that is needed for the construction of the the conformal measure on $L(G)$. However, we also need a third condition, which is automatically satisfied (see below) if $h$ is constructed as in [N].

$3^{\circ} . h(r+t) \leq h(r) h(t)$ for all positive $t$ and $r$.

We call a function $h$ satisfying these conditions Patterson function for $G$. A measure $\mu$ is a Patterson measure if it is a weak limit of measures $\mu_{i}$ obtained using a Patterson function, that is a weak limit of measures $\mu_{i}$ as in (5) below whose dimensions $\delta_{i}$ decrease to $\delta_{G}$.

If $h$ is constructed as in [N], p. 47, then $3^{\circ}$ easily follows. Nicholls follows Patterson $[\mathrm{P}]$ when constructing $h$ with the aid of an increasing sequence $X_{n}$ of positive numbers and another sequence $\varepsilon_{n}$ of decreasing positive numbers such that

$$
h_{N}(x)=h_{N}\left(X_{n}\right)\left(x / X_{n}\right)^{\varepsilon_{n}}
$$


if $X_{n} \leq x \leq X_{n+1}$. If $h(t)=h_{N}\left(e^{t}\right)$, then the derivative of $\log h(t)$ is $\varepsilon_{n}$ on $\left(X_{n}, X_{n+1}\right)$. Thus the derivative of $\log h(t)$ is non-increasing and $3^{\circ}$ follows.

We remark that while $3^{\circ}$ is a critical condition for us, only $1^{\circ}$ and $2^{\circ}$ are needed to show that a weak limit of measures $\mu_{i}$ in (3) is a conformal measure. Thus it is still possible that the measures which cannot be limits of Patterson measures, that is measures defined using $h$ as in $1^{\circ}-3^{\circ}$, could still be obtained by the Patterson construction using $h$ satisfying $1^{\circ}$ and $2^{\circ}$ but not $3^{\circ}$. However, the construction of $h$ in $[\mathrm{P}],[\mathrm{S}],[\mathrm{N}]$ is very natural and we doubt very much whether removing $3^{\circ}$ allows one to obtain every conformal measure as a weak limit of measures $\mu_{i}$ as in (3).

We remark that $3^{\circ}$ could be weakened in the sense that there is a constant $C$ not depending on $t$ and $r$ such that

$$
h(t+r) \leq C h(t) h(r) .
$$

Our arguments would still be valid with this weakened condition.

\section{Adapting the Shadow Lemma}

Let $G$ be a Kleinian group of $\mathbf{B}^{n}$ such that the Poincaré series of $G$ converges at the exponent of convergence $\delta_{G}$. Let $h$ be a Patterson function for $G$. We use $P_{h, \delta}$ as in (4) to define the atomic measures which, in the weak limit, give a conformal measure on $L(G)$.

Let $\delta>\delta_{G}$ and define the probability measure $\mu_{\delta}$ on $G z$ as

$$
\mu_{\delta}=\sum_{g \in G} c_{\delta} h(d(y, g(z))) e^{-\delta d(y, g(z))} \mu_{g(z)}
$$

where $\mu_{g(z)}$ is the atomic measure of mass 1 concentrated at $g(z)$ and the normalization constant $c_{\delta}=P_{h, \delta}(y, z)^{-1}$ is so chosen that the total mass is 1 . Note that the measure $\mu_{\delta}$ depends on the choice of $y$ and $z$.

We need estimates for $\mu_{\delta}$-measures of shadows of hyperbolic balls. If $z \in \mathbf{B}^{n}$ and $r>0$, let $S_{y}(z, r)$ be the shadow from $y \in \mathbf{B}^{n}$ of the hyperbolic disk $D(z, r)$ of hyperbolic radius $r$ and set $S(z, r)=S_{0}(z, r)$. Thus $w \in \overline{\mathbf{B}^{n}}$ is in $S_{y}(z, r)$ if the hyperbolic line segment or ray with endpoints $y$ and $w$ intersects $D(z, r)$. The following is an adaptation of Sullivan's Shadow Lemma.

Lemma. There is a constant $M$ depending on $r$ and $z$ (with $G$ fixed) such that if $\delta_{G}+1>\delta>\delta_{G}$ and $\zeta \in G z$, then

$$
\mu_{\delta}\left(S_{y}(\zeta, r)\right) \leq \operatorname{Mh}(d(y, \zeta)) e^{-\delta_{G} d(y, \zeta)} .
$$

Proof. We can assume that $y=0$ by conjugating $G$ with a Möbius transformation $g$ such that $g(y)=0$. We can also assume that $z=0$ (changing $z$ to 0 means only that we may need to multiply by a constant). Thus there is $g \in G$ such that $g(\zeta)=0$. The map $g$ transforms the shadow $S(\zeta, r)$ to the shadow 
$S_{g(0)}(0, r)=g(S(\zeta, r))$, that is $w \in g(S(\zeta, r))$ if the hyperbolic line segment or ray with endpoints $w$ and $g(0)$ intersects $D(0, r)$. Thus if $\gamma(0) \in G 0 \cap g(S(\zeta, r))$, then

$$
d(g(0), \gamma(0)) \leq d(g(0), 0)+d(0, \gamma(0)) \leq d(g(0), \gamma(0))+2 r .
$$

Since $h$ is increasing, it therefore follows that

$$
\begin{aligned}
\mu_{\delta}(S(\zeta, r)) & =\sum_{\gamma \in G, \gamma(0) \in S(\zeta, r)} c_{\delta} h(d(0, \gamma(0))) e^{-\delta d(0, \gamma(0))} \\
& =\sum_{\gamma \in G, \gamma(0) \in g S(\zeta, r)} c_{\delta} h(d(g(0), \gamma(0))) e^{-\delta d(g(0), \gamma(0))} \\
& \leq \sum_{\gamma \in G, \gamma(0) \in g S(\zeta, r)} c_{\delta} h(d(g(0), 0)+d(0, \gamma(0))) e^{-\delta(d(g(0), 0)+d(0, \gamma(0))-2 r)} .
\end{aligned}
$$

If we use $3^{\circ}$ and sum over all $\gamma \in G$, we obtain the following upper estimate for the last sum (recall that $\left.c_{\delta}=P_{h, \delta}(0,0)^{-1}\right)$ :

$$
\begin{aligned}
& h(d(g(0), 0)) e^{-\delta(-2 r+d(0, g(0)))} c_{\delta} \sum_{\gamma \in G} h(d(0, \gamma(0))) e^{-\delta d(0, \gamma(0))} \\
& =h(d(0, g(0))) e^{-\delta d(0, g(0))} e^{2 \delta r}=e^{2 \delta r} h(d(0, \zeta)) e^{-\delta d(0, \zeta)} .
\end{aligned}
$$

Thus the lemma is true with $M=e^{\left(2 \delta_{G}+2\right) r}$ if $z=0$.

\section{The main theorem}

Using the Shadow Lemma of Section 4, we can now obtain our main theorem. Recall that the $h$-modified Poincaré series of $G$ is written $\sum_{\gamma \in G} h(d(y, \gamma(z))) e^{-\delta d(y, \gamma(z))}$.

THEOREM. Let $G$ be a Kleinian group of $\mathbf{B}^{n}$ with exponent of convergence $\delta_{G}$. Suppose that the Poincaré series for $G$ converges at the exponent of convergence and let $h$ be a Patterson function for $G$. Let $E$ be an end of $G$, possibly with boundary, and let $G_{E}$ be the corresponding end group. Suppose that either $\delta_{G_{E}}<\delta_{G}$, or that $\delta_{G_{E}}=\delta_{G}$ and the h-modified Poincaré series of $G_{E}$ converges at the exponent $\delta_{G}=\delta_{G_{E}}$.

Let $\mu$ be a measure obtained by the Patterson-Sullivan construction using this Patterson function. Then $\mu\left(L_{e}(E)\right)=0$ and if the end is bounded $\mu\left(L\left(G_{E}\right)\right)=0$.

Proof. The proof is analogous to that of Theorem 4.4 of [AFT], using the adapted Shadow Lemma. We assume first that $E$ does not have boundary and indicate in the end the necessary changes for the case of ends with boundary.

Given $\delta>\delta_{G}$ and the Patterson function $h$, let $\mu_{\delta}$ be a measure defined by (5). For each $0<\rho<1$ we will define a neighbourhood $U_{\rho}$ of $L_{e}(E)$ in $\overline{\mathbf{B}^{n}}$ so that

$$
\mu_{\delta}\left(U_{\rho}\right) \leq M_{\rho}
$$


for a constant $M_{\rho}>0$ with the property that $M_{\rho} \rightarrow 0$ when $\rho \rightarrow 1$. This in turn will imply $\mu\left(L_{e}(E)\right)=0$. The details of the argument are given below.

We can assume without loss of generality that $0 \notin E$ and that $y=0$ (changing the basepoint $y$ does not change the measure class of $\mu$ ). Also, consider a point $z_{0} \in \partial E$ and assume $\mu$ is given by the $h$-modified Poincare series $\sum_{\gamma \in G} h\left(d\left(0, \gamma\left(z_{0}\right)\right)\right) e^{-\delta d\left(0, \gamma\left(z_{0}\right)\right)}$. Define $B_{\rho}=\left\{x \in \mathbf{B}^{n}:|x|>\rho\right\}$ and let $U_{\rho}$ be the union of all rays $R_{a}=\{t a: \rho<t \leq 1\}, a \in \mathbf{S}^{n-1}$, such that there is $\rho<t \leq 1$ with ta $\in E$. Thus $U_{\rho}$ is an open neighborhood of $L_{e}(E)$ in $\overline{\mathbf{B}^{n}}$.

Since $\partial E / G$ is compact and $z_{0} \in \partial E$, we can fix a number $r>0$ such that

$$
\bigcup_{\gamma \in G} D\left(\gamma\left(z_{0}\right), r\right) \supset \bigcup_{\gamma \in G} \gamma(\partial E) .
$$

As before, $D(z, r)$ is the open hyperbolic ball with center $z$ and radius $r$. Let $S_{\gamma}$ be the shadow of $D\left(\gamma\left(z_{0}\right), r\right)$ from 0 . Recall that $S_{\gamma}$ contains all the points $w \in \overline{\mathbf{B}^{n}}$ such that the hyperbolic line segment or ray with endpoints 0 and $w$ intersects $D\left(\gamma\left(z_{0}\right), r\right)$. Let now $V_{\rho}$ be the union of all shadows $S_{\gamma}, \gamma \in G_{E}$, such that $D\left(\gamma\left(z_{0}\right), r\right)$ intersects the set $B_{\rho}$ defined above. If a ray $R_{a}$ is contained in $U_{\rho}$, then either $R_{a} \subset E$ or $R_{a}$ intersects $\partial E$. In the latter case let $v$ be the point in $\mathbf{B}^{n}$ where $R_{a}$ meets $\partial E$ the first time (seen from $\rho a$ ), and let $R_{a}^{\prime}$ be the subray of $R_{a}$ which originates at $v$. Hence $R_{a}^{\prime}$ is contained in some $S_{\gamma}$ such that $D\left(\gamma\left(z_{0}\right), r\right)$ intersects $B_{\rho}$. It follows that

$$
G z_{0} \cap U_{\rho} \subset V_{\rho} .
$$

Next, we apply the Shadow Lemma of Section 4 to the measure $\mu_{\delta}$. Thus there exists a constant $M>0$ such that

$$
\mu_{\delta}\left(S_{\gamma}\right) \leq \operatorname{Mh}\left(d\left(0, \gamma\left(z_{0}\right)\right)\right) e^{-\delta_{G} d\left(0, \gamma\left(z_{0}\right)\right)}
$$

if $\delta_{G}+1>\delta>\delta_{G}$. Therefore we obtain for these $\delta$

$$
\sum \mu_{\delta}\left(S_{\gamma}\right) \leq \sum M h\left(d\left(0, \gamma\left(z_{0}\right)\right)\right) e^{-\delta_{G} d\left(0, \gamma\left(z_{0}\right)\right)}=: M_{\rho},
$$

where both sums are restricted to elements $\gamma \in G_{E}$ such that $D\left(\gamma\left(z_{0}\right), r\right)$ intersects $B_{\rho}$. Since the $h$-modified Poincaré series for $G_{E}$ converges at the exponent $\delta_{G}$, the numbers $M_{\rho}$ are finite and $M_{\rho} \rightarrow 0$ as $\rho \rightarrow 1$. The number $M_{\rho}$ is an upper bound for $\mu_{\delta}\left(V_{\rho}\right)$ if $\delta_{G}<\delta<\delta_{G}+1$. Since $G z_{0} \cap U_{\rho} \subset V_{\rho}, M_{\rho}$ is an upper bound for $\mu_{\delta}\left(U_{\rho}\right)$ as well.

Suppose now that $\mu$ is a Patterson measure obtained using this $h$, that is, suppose that $\mu$ is a weak limit of measures $\mu_{i}$ so that $\mu_{i}=\mu_{\delta_{i}}$ as in (5), and with $\delta_{i}$ decreasing to $\delta_{G}$. To see that $\mu\left(L_{e}(E)\right)=0$, let $\Lambda_{k}$ be the set of points $z \in \mathbf{S}^{n-1}$ such that the line segment $t z, t \in[1-1 / k, 1)$, is contained in $E \cup \partial E$. By construction, $U_{\rho}$ is a neighbourhood of $\Lambda_{k}$ for every $0<\rho<1$. Since all $\Lambda_{k}$ are closed, the inequalities $\mu_{i}\left(U_{\rho}\right) \leq M_{\rho}$ imply that $\mu\left(\Lambda_{k}\right) \leq M_{\rho}$ for all $\rho$ and hence $\mu\left(\Lambda_{k}\right)=0$. Finally, since $L_{e}(E)$ is contained in the union of the $\Lambda_{k}$, we conclude that $\mu\left(L_{e}(E)\right)=0$. 
If the end $E$ is bounded, then by [AFT] every $x \in L\left(G_{E}\right)$ is either an end limit point of $E$ or a conical limit point of $G_{E}$. Since the conical limit set has zero measure if the Poincare series converges at the dimension of the measure, it follows that $\mu\left(L\left(G_{E}\right)\right)=0$.

Finally, if the end $E$ is an end with boundary, then the above argument works if one replaces $\mathbf{B}^{n}$ with the hyperbolic convex hull $H_{G}$ of the limit set $L(G)$. We assume that $0 \in H_{G}$ and replace $\partial E$ by $\partial E \cap H_{G}$ on the right hand side of (6). Note that the $G$-quotient of $\partial E \cap H_{G}$ is compact and so there is such an $r_{0}$ as claimed in (6).

We say that $\mathscr{E}=\left\{E_{1}, \ldots, E_{p}\right\}$ is a complete collection of ends for $G$ if each $E_{i}$ is an end of $G$, the projections $p\left(E_{i}\right)$ of $E_{i}$ to $M_{G}$ are disjoint, and if $M_{G} \backslash\left(p\left(E_{1}\right) \cup \cdots \cup p\left(E_{p}\right)\right)$ is compact.

COROllary. Let $\mathscr{E}=\left\{E_{1}, \ldots, E_{p}\right\}$ be a complete collection of ends for $G$ and assume that the Poincare series of $G$ converges at the exponent of convergence $\delta_{G}$. Suppose $h$ is a Patterson function for $G$, and hence that the $h$-modified Poincaré series for $G$ diverges at $\delta_{G}$. Then there is an end $E \in \mathscr{E}$ such that the $h$-modified Poincaré series for $G_{E}$ diverges at $\delta_{G}$.

Proof. Let $\mu$ be a measure obtained by the Patterson construction. We know (see [AFT, Theorem 4.6]) that $\mu$ is supported by the union of endpoints of $g E_{i}$ where $g \in G$ and $i \leq p$. Hence $\mu\left(L_{e}\left(E_{i}\right)\right)>0$ for some $i$. By the preceding theorem, the $h$-modified Poincaré series for $G_{E_{i}}$ must diverge at $\delta_{G}$.

\section{Examples}

A. The first example was one of the main motivations for this work. Let $G$ be a Kleinian group acting on the hyperbolic $n$-space. Let $v$ be a parabolic fixed point of $G$. Then $G_{v}$ contains a free abelian subgroup of rank $k$ where $0<k<n$ in which case we say that $v$ has rank $k$. It is known that the exponent of convergence of $G_{v}$ in the rank $k$ case is $k / 2$ and that the Poincare series of $G_{v}$ diverges at exponent $k / 2$.

Assume that $v$ is a parabolic fixpoint of $G$ of rank $n-1$. Then there exists an open horoball $B$ at $v$ which is precisely invariant under $G_{v}$ in $G$, i.e. $\gamma(B)=B$ for all $g \in G_{v}$ and $\gamma(B) \cap B=\emptyset$ for all $\gamma \in G \backslash G_{v}$. Therefore, the stabilizers $G_{B}$ and $G_{v}$ of $B$ and $v$, respectively, coincide. Let $S^{0}$ be the boundary of $B$ in $\mathbf{B}^{n}$. Then $S=S^{0} / G=S^{0} / G_{v}$ is compact since $G_{v}$ has full rank. Thus $B$ is an end of $G$ with end group $G_{v}$, and $B / G$ is an end of $M_{G}$. As observed above, $\delta_{G_{v}}=(n-1) / 2$ and the Poincaré series for $G_{v}$ diverges. Hence, if the Poincaré series for $G$ converges at the exponent of convergence, then $\delta_{G}>\delta_{G_{v}}$ and so our Theorem implies that a Patterson measure for $G$ gives zero measure to endpoints of $B$, that is to the point $v$.

Let $v$ be an atomic measure with mass concentrated at $v$. Then $v$ is a conformal measure for $G_{v}$ for any dimension $\delta$, in particular, for $\delta=\delta_{G}$. Since 
the Poincare series for $G$ converges at the exponent $\delta$, the measure $v$ can be extended to a conformal measure for $G$ of dimension $\delta_{G}$ supported by the orbit $G v$, cf. [AFT, Proposition 4.5], and our Theorem shows that $v$ is not a Patterson measure.

Note that this also applies to bounded parabolic fixed points of rank $k<n-1$, since corresponding to such a fixed point $v$ there is a so-called cusp neighborhood of $V$ in $\overline{\mathbf{B}^{n}} \backslash L(G)$. This means that there is a Möbius transformation $h$ mapping the closed $n$-ball to the closed upper half-space of $\overline{\mathbf{R}^{n}}$, so that $h(v)=\infty$, the subspace $\mathbf{R}^{k}$ is invariant and has compact quotient with respect to the group $h G_{v} h^{-1}$, and $h(V)$ is the complement (in the closed upper half-space of $\mathbf{R}^{n}$ ) of a set of the form $\mathbf{R}^{k} \times \mathbf{B}^{n-k}$. If we project to the quotient, $\pi$ being the projection, then the boundary of $\pi(V)$ in $\bar{M}_{G}$ is compact and hence $\pi(V)$ is an end with boundary. We could see as above, applying the aforementioned results of [AFT] to ends with boundary, that $G v$ supports an atomic conformal measure which is not a Patterson measure.

Note that our Theorem can be regarded as a generalization and different proof of the fact that the Patterson construction gives a conformal measure which does not have atoms at bounded parabolic fixed points (see [N], Theorem 3.5.9). In order to obtain this property of Patterson measures, we only need to complement the above argument by the observation that if the Poincare series of $G$ diverges at $\delta_{G}$, then a Patterson measure for $G$ does not have atoms (see $[\mathrm{N}]$, Theorem 3.5.8).

B. The second example is more complex. Let $G$ be a geometrically infinite, topologically tame Kleinian group of the second kind acting on $\mathbf{B}^{3}$, for instance a simply degenerate surface group (for the definition see for instance [MT]). Then it is known that the Poincaré series converges at the exponent of convergence which is equal to 2 (cf. [C]). Let $F$ be a fundamental domain for $G$ acting on the boundary sphere. Let $D, D_{1}, \ldots, D_{4}$ be five closed disks contained in the interior of $F$ such that $D_{1}, \ldots, D_{4}$ are disjoint and contained in the interior of $D$. Let $h$ and $g$ be two loxodromic elements so that $h\left(\partial D_{1}\right)=\partial D_{2}$ and $g\left(\partial D_{3}\right)=\partial D_{4}$, and so that $h$ and $g$ generate a Schottky group $H$ whose fundamental domain $F^{\prime}$ is the closure of the complement of $D_{1} \cup D_{2} \cup D_{3} \cup D_{4}$. (Here, $\partial$ denotes the topological boundary in the boundary sphere of $\mathbf{B}^{3}$.) Thus $H$ is geometrically finite and hence $\delta_{H}<2$. Let $N$ be the normalizer of $h$ in $H$, making $N$ an infinitely generated Kleinian group such that $\delta_{N} \leq \delta_{H}<2$. (In fact, by a result of M. Rees [R] we even have that $\delta_{N}=\delta_{H}$.) The group $N$ is infinitely generated and $h_{i}=g^{i} h g^{-i}$ are free generators. It has a fundamental domain $D_{N}$ contained in the closure of the complement of the union of all $g^{i}\left(D_{1}\right) \cup g^{i}\left(D_{2}\right)$.

Let now $\Gamma_{0}$ be the group generated by $H$ and $G$. The group $\Gamma_{0}$ is Kleinian, its fundamental domain is $F \cap F^{\prime}$ and $\Gamma_{0}$ is the free product $H * G$. Therefore, the subgroup $\Gamma=N * G$ of $\Gamma_{0}$ is also Kleinian. The group $\Gamma$ is the example we are seeking.

Let $S$ be a hyperbolic subplane of $\mathbf{B}^{3}$ bounded by $\partial D$. Then $\gamma S, \gamma \in N$, are distinct and it is easy to see that they bound an end with boundary of $\Gamma$, denoted by $E$, whose end group $G_{E}$ is just $N$. We note that this end is bounded. To 
see this, let $F$ be the component of $\overline{\mathbf{B}^{3}} \backslash S$ not intersecting $E$. Then the intersection of $\bar{F}$ with the boundary sphere of $\mathbf{B}^{3}$ is contained in the interior of the fundamental domain $D_{N}$ of the action of $N$ on the boundary sphere. Hence $g \bar{F}, g \in N=G_{E}$, is a family of disjoint sets. This fact implies that $\bar{F}$ projects homeomorphically onto a subset of $\left(\overline{\mathbf{B}^{3}} \backslash L\left(G_{E}\right)\right) / G_{E}$ which is the complement of $E / G_{E}$. Thus the complement of $E / G_{E}$ in $M_{G_{E}}$ is compact and it follows that $E$ is a bounded end. Hence $L\left(G_{E}\right)$ is the union of conical limit points of $G_{E}$ and of the end limit points of $E$, cf. [AFT, Lemma 3.1].

If $L\left(G_{E}\right)$ would consist of conical limit points only, then $G_{E}$ would be a convex cocompact group and hence finitely generated. Since $G_{E}$ is not finitely generated we can conclude that the set of end limit points $L_{e}(E)$ is not empty. We have that $2 \geq \delta_{\Gamma} \geq \delta_{G}=2$ and hence $\delta_{\Gamma}=2$. Since $\Gamma$ is of the second kind, the Poincare series of $\Gamma$ converges at $\delta_{\Gamma}=2$. Hence there is a conformal measure $v$ of $N$ supported by $L_{e}(E)$, cf. Theorem 4.4 of [AFT]; this theorem, like the next one to which we refer, is formulated for ends without boundary but is valid also for ends with boundary (see the discussion in the end of Section 5 of $[\mathrm{AFT}])$. We can extend $v$ to a conformal measure of $\Gamma$ supported by $\bigcup_{g \in \Gamma} g\left(L_{e}(E)\right)$, cf. [AFT, Theorem 4.7]. Since $\delta_{N}=\delta_{G_{E}}<2=\delta_{\Gamma}$, our Theorem implies that $v$ is not a Patterson measure.

\title{
REFERENCES
}

[AFT] J. W. Anderson, K. FAlk AND P. Tukia, Conformal measures associated to ends of hyperbolic $n$-manifolds, preprint (arXiv: math.CV/0409582).

[C] R. D. CAnary, On the Laplacian and the geometry of hyperbolic 3-manifolds, J. Diff. Geom. 36 (1992), 349-367.

[MT] K. MatsuZaki and M. Taniguchi, Hyperbolic manifolds and Kleinian groups, Clarendon Press, Oxford, 1998.

[N] P. J. Nicholls, The ergodic theory of discrete groups, London Mathematical Society lecture notes series 143, Cambridge University Press, 1989.

[P] S. J. Patterson, The limit set of a Fuchsian group, Acta Math. 136 (1976), 241-273.

[R] M. REES, Checking ergodicity of some geodesic flows with infinite Gibbs measure, Ergod. Th. and Dynam. Sys. 1 (1981), 107-133.

[S] D. Sullivan, The density at infinity of a discrete group of hyperbolic motions, Inst. Hautes Études Sci. Publ. Math. 50 (1979), 171-202.

\author{
Department of Mathematics \\ Nui MAYNOOTH \\ Co. Kildare \\ IRELAND \\ E-mail: kfalk@maths.nuim.ie \\ Department of Mathematics and Statistics \\ P. O. Box 68 (Gustaf Hällströmin Katu 2B) \\ FI-00014 UNIVERSITY OF HeLSINKI \\ FINLAND \\ E-mail: pekka.tukia@helsinki.fi
}

